\title{
QUERATOPLASTIA ENDOTELIAL DE MEMBRANA DE DESCEMET (DMEK): RESULTADOS A DOS AÑOS
}

\author{
DESCEMET MEMBRANE ENDOTHELIAL KERATOPLASTY \\ (DMEK): TWO-YEAR RESULTS
}

\author{
DAPENA I ${ }^{1}$, HAM L ${ }^{2}$, LIE J ${ }^{3}$, VAN-DER-WEES J ${ }^{3}$, MELLES GRJ ${ }^{4}$
}

\begin{abstract}
RESUMEN
Objetivo: Describir los resultados, dos años después de realizar una queratoplastia endotelial de membrana de Descemet (DMEK: Descemet membrane endothelial keratoplasty), para el tratamiento de alteraciones del endotelio corneal.

Métodos: Estudio clínico prospectivo no randomizado. En 10 pacientes con distrofia endotelial de Fuchs o queratopatía bullosa, se practicó una DMEK. A través de una incisión de 3,5 mm en córnea clara, la membrana de Descemet (MD) del receptor fue desprendida del estroma posterior en presencia de aire. Un disco de $9 \mathrm{~mm}$ de diámetro enrollado de MD donante preservada, fue insertado en la cámara anterior del receptor, posicionado en contacto con el estroma posterior corneal y asegurado en su posición mediante el llenado completo de la cámara anterior con aire durante 30 minutos.

Resultados: Tres ojos mostraron un desprendimiento completo del tejido donante, por lo que fueron sometidos posteriormente a una queratoplastia endotelial con «pelado» de la MD (DSEK: Desce-
\end{abstract}

\begin{abstract}
Purpose: To report the two-year results of Descemet membrane endothelial keratoplasty (DMEK) for managing corneal endothelial disorders.

Methods: Non-randomized prospective clinical trial. A DMEK was performed in ten patients with Fuchs' endothelial dystrophy or bullous keratopathy. A $3.5 \mathrm{~mm}$ clear corneal incision was made and «under air» DM was stripped off from the posterior stroma. A $9.0 \mathrm{~mm}$ diameter, organ cultured donor DM roll was inserted into a recipient anterior chamber, positioned into the posterior stroma and secured by completely filling the anterior chamber with air for 30 minutes.

Results: Three eyes showed complete detachment of the tissue; this was managed by a secondary Descemet stripping endothelial keratoplasty procedure. The remaining seven eyes had a best corrected visual acuity of $\geq 0.7$ in three eyes (43\%) at one month, in five eyes $(71 \%)$ at six months, and in six eyes $(86 \%)$ at one and two years. At six months, the endothelial cell density averaged $2039( \pm 373)$
\end{abstract}

Recibido: 15/10/08. Aceptado: 24/4/09.

Netherlands Institute for Innovative Ocular Surgery. Rotterdam. The Netherlands.

${ }^{1}$ Licenciado en Medicina. Netherlands Institute for Innovative Ocular Surgery, Rotterdam, Holanda. Melles Cornea Clinic Rotterdam, Rotterdam, Holanda.

${ }^{2}$ Licenciado en Ciencias de la Salud. Netherlands Institute for Innovative Ocular Surgery, Rotterdam, Holanda. Melles Cornea Clinic Rotterdam, Rotterdam, Holanda.

${ }^{3}$ Licenciado en Ciencias de la Salud. Doctor en Ciencias de la Salud. Netherlands Institute for Innovative Ocular Surgery, Rotterdam, Holanda. Amnitrans EyeBank Rotterdam, Rotterdam, Holanda.

${ }^{4}$ Licenciado en Medicina. Doctor en Medicina. Netherlands Institute for Innovative Ocular Surgery, Rotterdam, Holanda. Melles Cornea Clinic Rotterdam, Rotterdam, Holanda. Amnitrans EyeBank Rotterdam, Rotterdam, Holanda.

Correspondencia:

Gerrit R.J. Melles, MD, PhD

Netherlands Institute for Innovative Ocular Surgery

Laan Op Zuid, 88

3071 AA Rotterdam

The Netherlands

E-mail: melles@niioc.nl / isadapena@gmail.com 
met stripping endothelial keratoplasty). En los siete ojos restantes, se observó una agudeza visual mejor corregida (AVMC) $\geq$ a 0,7 en 3 ojos (43\%) en el primer mes, en 5 ojos (71\%) a los seis meses, y en seis ojos $(86 \%)$ al primer y segundo años. A los seis meses, la densidad celular endotelial media fue de 2039 (DS: 373) cél/ $/ \mathrm{mm}^{2}$ ( $\mathrm{n}=7$ ), al año de 1925 (DS: 267 cél $/ \mathrm{mm}^{2}(\mathrm{n}=7)$ y a los 2 años de 1730 (DS: 400) cél $/ \mathrm{mm}^{2}(\mathrm{n}=6)$.

Conclusión: DMEK podría proporcionar una recuperación rápida y casi completa de la visión. Debido a que el tejido donante puede ser obtenido a partir de anillos córneo-esclerales donantes, el procedimiento podría ser fácilmente accesible para la mayoría de los cirujanos corneales.

Palabras clave: Queratoplastia endotelial de membrana de Descemet (DMEK), queratoplastia lamelar posterior, transplante corneal, membrana de Descemet, endotelio, técnica quirúrgica. cells $/ \mathrm{mm}^{2}(\mathrm{n}=7)$, at one year $1925( \pm 267)$ cells $/ \mathrm{mm}^{2}$ $(\mathrm{n}=7)$ and at two years $1730( \pm 400)$ cells $/ \mathrm{mm}^{2}$ $(\mathrm{n}=6)$.

Conclusions: DMEK may provide quick and nearly complete visual rehabilitation. Since the donor tissue can be stripped from donor corneoscleral rims, the procedure may be readily accessible to most corneal surgeons (Arch Soc Esp Oftalmol 2009; 84: 237-244).

Key words: Descemet membrane endothelial keratoplasty (DMEK), posterior lamellar keratoplasty, corneal transplantation, Descemet membrane, endothelium, surgical technique.

\section{INTRODUCCIÓN}

En los últimos años hemos descrito varios procedimientos de queratoplastia lamelar posterior que permiten la sustitución del endotelio corneal sin incisiones ni suturas en la superficie corneal, para el manejo de la distrofia endotelial de Fuchs y de la queratopatía bullosa afáquica o pseudofáquica. En 1998, describimos una técnica en la que un disco lamelar posterior sin suturas, de $7,5 \mathrm{~mm}$ de diámetro, podía ser transplantado a través de una incisión escleral de $9,0 \mathrm{~mm}$ suturada posteriormente (1-3). Desde 2001, la técnica se popularizó en Estados Unidos como «Queratoplastia endotelial lamelar profunda» (DLEK: Deep lamellar endotelial keratoplasty) (4). En el 2000, describimos otra técnica sin requerimiento de suturas, modificación de la previa, en la que un trasplante posterior en forma de disco de 9,0 a $9,5 \mathrm{~mm}$ de diámetro, era insertado a través de una incisión escleral tunelizada autosellable de $5,0 \mathrm{~mm}$ y desplegado en la cámara anterior (5). Desde 2005, esta técnica ha sido popularizada en Estados Unidos como «DLEK de pequeña incisión» (6). En el 2003, describimos la inserción de un disco posterior donante plegado en combinación con una «descemetorrexis» para retirar la capa endotelial y su MD del receptor $(7,8)$. Esta última técnica es denominada actualmente queratoplastia endotelial con «pelado» de la membrana de Descemet (Descemet's stripping endotelial keratoplasty: DSEK) $(9,10)$.

A pesar de que las diversas técnicas diseñadas y desarrolladas por nuestro Instituto demostraron ser viables para el trasplante de un disco corneal posterior donante sin suturas, cabría esperar que el trasplante selectivo de MD y endotelio proporcionase la mejor recuperación posible de la capacidad visual en una córnea con alteración endotelial (1115). El presente estudio describe los resultados clínicos a los dos años del trasplante de MD aislada, a través de una incisión autosellable de 3,5 $\mathrm{mm}$ en córnea clara, que podría ser denominada «Queratoplastia Endotelial de Membrana de Descemet» (Descemet Membrane Endothelial Keratoplasty: DMEK) (13-15).

\section{SUJETOS, MATERIAL Y MÉTODOS}

La DMEK fue realizada en cuatro hombres y en seis mujeres, de 45 a 87 años de edad con distrofia endotelial de Fuchs y/o queratopatía bullosa (tabla I). Todos los pacientes firmaron un consentimiento informado aprobado por el IRB (Institutional Review Board). 
Tabla I. Queratoplastia endotelial de membrana de Descemet (DMEK): Resultados a 2 años

\begin{tabular}{|c|c|c|c|c|c|c|c|c|c|c|c|c|c|c|c|c|c|}
\hline \multicolumn{4}{|c|}{ Paciente } & \multicolumn{3}{|c|}{ Prequirúrgico } & \multirow{3}{*}{$\begin{array}{c}\text { Donante } \\
\text { DCE } \\
(\mathrm{cel} /) \\
\mathrm{mm}^{2}\end{array}$} & \multirow{3}{*}{$\frac{\text { Cirugía }}{\text { Complicaciones }}$} & \multicolumn{9}{|c|}{ Postquirúrgico } \\
\hline \multirow[t]{2}{*}{ \# } & \multirow[t]{2}{*}{$\begin{array}{l}\text { Edad } \\
\text { (años) }\end{array}$} & \multirow[t]{2}{*}{ Sexo } & \multirow[t]{2}{*}{$\begin{array}{l}\text { OD/ } \\
\text { OS }\end{array}$} & \multirow[t]{2}{*}{$\begin{array}{l}\text { Indicación } \\
\text { de cirugía }\end{array}$} & \multirow[t]{2}{*}{$\begin{array}{l}\text { Enfermedad } \\
\text { concomitante }\end{array}$} & \multirow[t]{2}{*}{ AVMC } & & & \multicolumn{4}{|c|}{ AVMC } & \multirow[t]{2}{*}{$\mathrm{BMC}$} & \multicolumn{3}{|c|}{$\begin{array}{l}\mathrm{DCE} \\
\left(\mathrm{cel} / \mathrm{mm}^{2}\right)\end{array}$} & \multirow[t]{2}{*}{ Observaciones } \\
\hline & & & & & & & & & $1 \mathrm{~m}$ & $6 \mathrm{~m}$ & $12 \mathrm{~m}$ & $24 \mathrm{~m}$ & & $6 \mathrm{~m}$ & $12 \mathrm{~m}$ & $24 \mathrm{~m}$ & \\
\hline & 64 & M & OS & $\begin{array}{l}\text { FED } \\
\text { (pseudofáquico) }\end{array}$ & Ninguna & 0,3 & 2960 & No & 1,0 & 1,25 & 1,0 & 1,0 & Adherido & 1750 & 1678 & 1608 & Ninguna \\
\hline & 61 & $\mathrm{~F}$ & OD & PPBK & Ninguna & $\mathrm{CD}$ & 2630 & $\begin{array}{l}\text { Inserción del } \\
\text { injerto } \\
\text { complicada }\end{array}$ & n.r. & n.r. & n.r. & n.r. & n.r. & n.r. & n.r. & n.r. & $\begin{array}{l}\text { DSEK } \\
\text { no complicada } \\
2 \text { semanas } \\
\text { tras la DMEK } \\
\text { inicial }\end{array}$ \\
\hline & 87 & $\mathrm{~F}$ & OS & PPBK & $\begin{array}{l}\text { Pupila inmóvil } \\
\text { y cambios del } \\
\text { EPR tras faco } \\
\text { complicada }\end{array}$ & 0,1 & 2460 & No & 0,25 & 0,4 & 0,4 & n.d. & Adherido & 1760 & 1675 & n.d. & Ninguna \\
\hline & 86 & $\mathrm{~F}$ & OD & $\begin{array}{l}\text { FED } \\
\text { (pseudofáquico) }\end{array}$ & $\begin{array}{l}\text { Cicatriz estromal } \\
\text { superficial }\end{array}$ & 0,3 & 2640 & Presión vítrea & n.r. & n.r. & n.r. & n.r. & n.r. & n.r. & n.r. & n.r. & $\begin{array}{l}\text { DSEK } \\
\text { no complicada } \\
3 \text { semanas tras } \\
\text { la DMEK } \\
\text { inicial }\end{array}$ \\
\hline & 72 & M & OD & $\begin{array}{l}\text { FED } \\
\text { (pseudofáquico) }\end{array}$ & $\begin{array}{l}\text { Cicatriz corneal } \\
\text { central subepitelial }\end{array}$ & 0,1 & 2660 & $\begin{array}{l}\text { Posición donante } \\
\text { excéntrica }\end{array}$ & 0,5 & 0,6 & 0,7 & 0,7 & $\begin{array}{l}\text { Adherido; } \\
\text { pequeño } \\
\text { DM roll } \\
\text { paracentral }\end{array}$ & 1790 & 1812 & 1171 & Ninguna \\
\hline & 60 & M & OS & $\begin{array}{l}\text { FED } \\
\text { (pseudofáquico) }\end{array}$ & Ninguna & 0,3 & 2470 & No & 1,0 & 1,0 & 1,0 & 1,0 & Adherido & 2270 & 2100 & 1800 & Ninguna \\
\hline 7 & 76 & $\mathrm{~F}$ & OS & $\begin{array}{l}\text { FED } \\
\text { (pseudofáquico) }\end{array}$ & Ninguna & 0,3 & 2520 & No & 0,6 & 0,8 & 1,0 & 0,9 & Adherido & 2180 & 2369 & 2289 & Ninguna \\
\hline 8 & 59 & M & OD & BK eci (fáquico) & Ninguna & 0,8 & 2480 & No & 1,0 & 1,0 & 1,0 & 1,0 & Adherido & 1790 & 1743 & 1476 & Ninguna \\
\hline 9 & 74 & $\mathrm{~F}$ & OS & $\begin{array}{l}\text { FED } \\
\text { (pseudofáquico) }\end{array}$ & Ninguna & 0,3 & 2640 & No & 0,6 & 0,7 & 0,8 & 1,0 & Adherido & 2730 & 2098 & 2034 & Ninguna \\
\hline 10 & 45 & $\mathrm{~F}$ & OD & FED (fáquico) & LES & 0,5 & 2640 & $\begin{array}{l}\text { Desdoblamiento } \\
\text { donante } \\
\text { incompleto }\end{array}$ & n.r. & n.r. & n.r. & n.r. & n.r. & n.r. & n.r. & n.r. & $\begin{array}{l}\text { DSEK } \\
\text { no complicada } \\
3 \text { semanas tras } \\
\text { la DMEK } \\
\text { inicial }\end{array}$ \\
\hline
\end{tabular}

DMAE= Degeneración macular asociada a la edad; DSEK = Descemet stripping endothelial keratoplasty; $\mathrm{CD}=$ Cuenta dedos; n.r. = No relevante; AVMC = Agudeza visual mejor corregida (Snellen); DCE= Densidad celular endotelial; PPBK = Queratopatía bullosa pseudofáquica; LES = Lupus eritematoso sistémico; MD= Membrana de Descemet; FED = Distrofia endotelial de Fuchs; EPR $=$ Epitelio pigmentario de la retina; $\mathrm{BMC}=$ Biomicroscopía $; \mathrm{BK}$ eci $=$ Queratopatia bullosa de origen desconocido; $\mathrm{m}=$ Meses; n.d. = No disponible

\section{Tejido donante}

En diez globos oculares donantes de menos de 36 horas post mortem, se extrajeron los anillos córneoesclerales y fueron almacenados en un medio esencial mínimamente modificado (EMEM) a $31^{\circ} \mathrm{C}$ para cultivo de órganos. La edad media de los donantes fue de 71,3 años (DS: 6,1) y el recuento endotelial celular medio del tejido fue de 2614 (DS: 186) $\mathrm{cel} / \mathrm{mm}^{2}$ (tabla).

Tras dos semanas en medio de cultivo sin dextrano y con antibióticos a $31^{\circ} \mathrm{C}$, se evaluó la morfología y viabilidad de las células endoteliales mediante microscopía invertida (Axiovert 40, Zeiss, Göttingen, Germany). Posteriormente, los anillos córneo-esclerales se montaron con la cara endotelial hacia arriba sobre un instrumento de sujeción por vacío de diseño propio y la MD fue despegada del estroma posterior mediante el uso de pinzas finas, obteniéndose una monocapa endotelial de 9,0 mm de diámetro de MD. Debido a las propiedades elásticas de la membrana de Descemet, ésta se enrolló espontáneamente formando un «rollo» de Descemet («Descemet-roll»), con el endotelio en su cara externa. Cada «Descemet-roll» fue almacenado posteriormente en un medio de cultivo de órganos sin dextrano y con antibióticos, durante un tiempo máximo de una semana, hasta el momento del trasplante.

\section{Cirugía}

En los ojos receptores, se realizó una marca epitelial de 9,0 mm de diámetro para delimitar el área de escisión de la MD. En el limbo se practicó una incisión tunelizada de $3,5 \mathrm{~mm}$, penetrando en la cámara anterior justo en el límite de dicha marca. 
Con un «rascador» de diseño propio (Melles scraper, D. O. R. C. International, Zuidland, The Netherlands) se desprendió una porción circular de MD del estroma posterior en presencia de aire, realizándose una «descemetorrexis» de 9,0 mm de diámetro y retirándose del ojo la porción central de la misma $(7,8)$.

El «Descemet-roll» donante fue teñido con una solución de azul tripán al 0,06\% (VisionBlue ${ }^{\mathrm{TM}}$, D. O. R. C. International) y cargado en un inyector de diseño propio (Hippocratech, Rotterdam, The Netherlands) (11,12). Utilizando el inyector, el «Descemet-roll» donante fue insertado en la cámara anterior y extendido cuidadosamente sobre la superficie iridiana mediante la manipulación indirecta del tejido con aire y solución salina balanceada. A continuación, se inyectó una burbuja de aire debajo de la MD donante para posicionarla y mantener el tejido en contacto con el estroma posterior del receptor $(11,12)$. La cámara anterior se rellenó completamente con aire durante 30 minutos, seguido de un recambio aire-líquido para presurizar el ojo.

\section{Evaluación del endotelio}

La viabilidad celular del endotelio donante se evaluó mediante microscopía de luz invertida (Axiovert 40, Zeiss, Göttingen, Germany), realizándose fotografías digitales (PixeLINK PL-A662, Zeiss, Göttingen, Germany) tras la instilación de sucrosa al 1,8\% y tinción con azul tripán al 0,04\% (15-17).

El endotelio de los pacientes fue fotografiado y evaluado empleando un microscopio especular Topcon SP3000p con autofoco de no-contacto (Topcon Corp, Tokyo, Japan). Las imágenes de la córnea central fueron analizadas y corregidas manualmente y se promediaron tres medidas de la densidad celular endotelial $(15-17,19,20)$.

\section{RESULTADOS}

En el primer día del postoperatorio, todas las MD donantes mostraron una adhesión completa al estroma posterior del receptor. A la semana, el trasplante de Descemet se mantuvo en posición en siete de los ojos; pero en los tres ojos restantes (casos 2, 4 y $10)$, se produjo un desprendimiento completo de la membrana donante, observándose el injerto enrollado y flotando en la cámara anterior. En estos ojos, se practicó una DSEK sin complicaciones, dos a tres semanas después de la cirugía inicial de DMEK.

En los siete ojos que mostraron una adhesión completa del injerto, se observó una agudeza visual mejor corregida (AVMC) $\geq$ a 0,7 en tres de los ojos (43\%) al primer mes de la cirugía, en cinco ojos (71\%) a los seis meses y en seis ojos (86\%) al primer y segundo años (tabla I). A los seis meses, la densidad celular endotelial media fue de 2039 (DS: 373) cel $/ \mathrm{mm}^{2}(\mathrm{n}=7)$ y al primer y segundo años de 1925 (DS: 267) cél $/ \mathrm{mm}^{2}$ (n=7) y de 1730 (DS: 400) cél $1 / \mathrm{mm}^{2}$ respectivamente (fig. 1 , tabla I).

Al principio, la visualización del trasplante en el ojo receptor fue difícil. La confirmación de que el trasplante se encontraba en posición, fue obtenida mediante la observación de su contorno periférico por medio de un «brillo» reflectante sobre la MD donante y mediante la microscopía especular identificatoria de las células donantes endoteliales paracentrales. En algunos ojos se observaron pequeñas «extensiones» periféricas de MD («tags» de Descemet), indicando la presencia de microdesprendimientos o pliegues de MD en el límite externo del trasplante. Con el tiempo esos «tags» periféricos se estabilizaron y no aumentaron de tamaño.

\section{DISCUSIÓN}

En 1998 describimos que el trasplante de MD era técnicamente posible en un modelo de ojo humano de cadáver $(11,12)$. En aquellos momentos, obtener MD a partir de un anillo córneo-escleral donante, era considerado un desafío sin el apoyo de un banco de ojos. Ya que el pelado de la MD podía ser en ocasiones complicado debido al desgarro inadvertido de la membrana, la MD donante podía ser preparada preferentemente en el banco de ojos, previo a la cirugía, mejor que como parte de la intervención misma. Con el inicio del Banco de ojos Amnitrans en Rotterdam (Amnitrans Eyebank Rotterdam) en enero de 2004, todas sus instalaciones y logística pudieron ser empleadas para la preparación rutinaria del trasplante de Descemet, la evaluación de su capa de células endoteliales y para el estudio microbiológico del tejido que asegurase su esterilidad. Desde entonces, estudios de laboratorio más extensos fueron realizados para simplificar el procedi- 

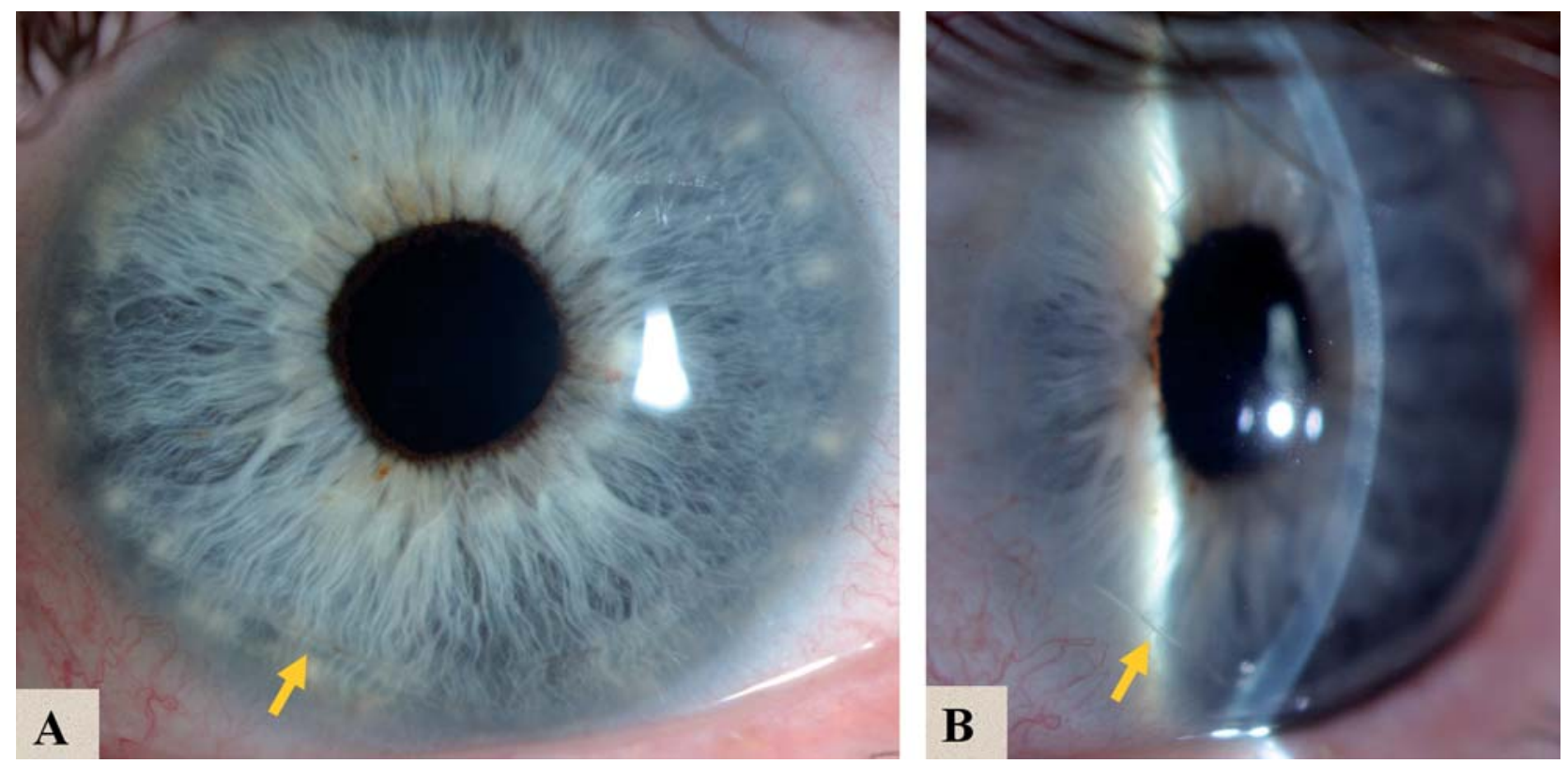

Fig. 1: Fotografía en lámpara de hendidura de una córnea transplantada un año después de realizar una DMEK (Caso 6). A) Obsérvese que la córnea trasplantada es transparente y con una interfase anatómica invisible entre los tejidos donante y huésped. B) Nótese el extremo periférico del trasplante de membrana de Descemet señalado por la flecha amarilla.

miento (14-18). Además, se diseñaron varias modificaciones técnicas para la implantación quirúrgica de la capa de MD donante, su manipulación en la cámara anterior y su adherencia completa al estroma posterior receptor. (Melles CRJ, unpublished data, 2005) $(14,15)$.

Recientemente, la posibilidad de transplantar una MD aislada fue mostrada también por Tappin y Pavel empleando distintos enfoques. Tappin diseñó un instrumento para insertar una MD donante de 7,5 mm de diámetro en la cámara anterior a través de una incisión de 8,0 mm suturada (21). Pavel describió el trasplante de tejido donante de MD ligado a un anillo periférico estromal para facilitar el manejo del mismo (P. Studeny. Descemet's membrane with stromal hem transplantation. Winter ESCRS 2007). En el presente estudio, trasplantamos MD con endotelio a través de una pequeña incisión tunelizada corneal como ha sido descrito previamente $(11,12)$. Teniendo en cuenta la nomenclatura utilizada para distinguir distintos procedimientos de queratoplastia lamelar posterior, el procedimiento actualmente descrito, es decir el trasplante de MD donante aislado portando una capa celular endotelial viable, fue inicialmente denominado DMEK.
El desafío al diseñar cualquier procedimiento de queratoplastia lamelar posterior, es proporcionar una densidad celular endotelial suficientemente alta como para obtener una supervivencia del injerto de larga duración. En el presente estudio, ambas medidas objetivas y subjetivas indicaron que la DMEK, podría tener potencial para conseguir densidades celulares próximas a 2000 células $/ \mathrm{mm}^{2}$ a medio plazo, comparables a aquellas obtenidas tras DLEK y DSEK (6-19).

En comparación con las técnicas de queratoplastia lamelar posterior previamente diseñadas por nosotros como DLEK y DSEK (14), DMEK podría tener cinco grandes ventajas. En primer lugar, el presente estudio sugiere que la recuperación visual tras DMEK podría ser mucho más rápida que tras DLEK o DSEK, ya que seis de siete ojos con una DMEK consiguieron una AVMC $\geq$ a 0,5 al mes de la cirugía (tabla I). Además, en la primera serie descrita de 50 pacientes sometidos a DMEK por distrofia endotelial de Fuchs, el 95\% presentó una AVMC $\geq$ a $0,50,5$, y lo que es más importante, el $75 \%$ alcanzó una AVMC $\geq$ a 0,8 a los 6 meses de la cirugía (22). En contraste con los procedimientos DLEK o DSEK, que han demostrado obtener una AVMC media $\geq$ a 0,5 en un tiempo medio de seis o más meses $(6,9,23)$. Esta diferencia en la recupera- 
ción de agudeza visual podría sugerir que es la presencia de estroma posterior donante en DLEK y DSEK, más que cambios secundarios o edema en el estroma corneal receptor, lo que determinaría primariamente el grado de recuperación visual, ya que no hay estroma posterior donante trasplantado en DMEK.

No obstante, para poder realizar una DMEK, es necesaria la visualización adecuada de la cámara anterior dada la mayor complejidad quirúrgica del procedimiento; por lo que en córneas gravemente descompensadas, en presencia de dispositivos de drenaje para glaucoma, cámara anterior estrecha o bajo potencial para rehabilitación visual, seria recomendable la realización de una DSEK, cirugía de menor dificultad técnica.

En segundo lugar, ya que la DMEK podría proporcionar una casi perfecta restauración de la anatomía corneal y una mejor calidad de la córnea, cabría esperar una mayor recuperación de la capacidad visual con esta técnica $(11,12)$. Por ello, en la DMEK, el resultado visual final podría estar limitado exclusivamente por la calidad de la porción anterior de la córnea del receptor previo a la cirugia.

En la distrofia endotelial de Fuchs, no es infrecuente que la agudeza visual no se correlacione con los síntomas visuales del paciente, por lo que, en estos casos, no siempre sería ésta un buen indicador para la cirugía. Ejemplo de esto sería el caso número 8 , que presentaba una AVMC de 0,8 , pero una córnea ampliamente descompensada, con cambios secundarios subepiteliales, y una alteración significativa de la sensibilidad al contraste y de la percepción de los colores.

En tercer lugar, en la DMEK, el diámetro del injerto puede ser de 9,0 a $11,0 \mathrm{~mm}$, por lo que es trasplantada una superficie celular endotelial mayor en comparación con DLEK (7,5 a 8,0 mm) o DSEK $(8,5$ a $9,5 \mathrm{~mm})$. En nuestra experiencia, aunque no haya sido demostrada la correlación entre el diámetro del injerto y su supervivencia; cabría esperar que un injerto mayor en DMEK, beneficiase la supervivencia del trasplante a largo plazo.

En cuarto lugar, la DMEK podría cumplir mejor los requerimientos actuales de una cirugía moderna del segmento anterior, ya que la MD donante puede ser trasplantada a través de una incisión tunelizada en córnea clara, que es ampliamente utilizada en la cirugía de facoemulsificación, y es conocido que induce un astigmatismo postoperatorio mínimo $(17,24,25)$. A pesar de que han sido descritos varios instrumentos para la inserción de la MD donante $(21,26,27)$, su utilización podría limitar el diámetro final del injerto $(6,0$ a 7,5 mm), además de requerir una incisión de entrada relativamente amplia.

En quinto lugar, en contraste con DLEK y DSEK, para las cuales la mayor parte de los cirujanos necesitarían un microqueratomo o láser de femtosegundo, las láminas de MD donante pueden ser «peladas» directamente a partir de un anillo córneo-escleral en DMEK. Con nuestra técnica para la preparación de láminas de 9,0 mm de MD a partir de anillos córneo-esclerales donantes cóncavos, la magnitud de la lesión celular expresada en el 'porcentaje de área de superficie endotelial media lesionada' es de $3,4 \% \quad(11,12,18)$. Resultados similares fueron encontrados por Ignacio et al, que obtuvieron trasplantes de Descemet a partir de anillos convexos (28), y Zhu et al, que preservaron láminas rectangulares a partir de anillos cóncavos (29). Es importante destacar, que todos los estudios se realizaron en tejidos preservados, bien por almacenamiento en frío o en medio de cultivo de órganos. Por lo tanto, si es posible obtener rutinariamente injertos viables a partir de tejidos preservados, la DMEK podría ser mucho más accesible para la mayor parte de los cirujanos corneales que la DLEK o la DSEK.

Al igual que en DSEK/DSAEK, el desprendimiento del trasplante destacó como la complicación más frecuente de la DMEK. En la primera serie descrita de 50 pacientes sometidos a DMEK por distrofia endotelial de Fuchs, la frecuencia de desprendimiento fue de un $25 \%$, no obstante, al analizar los últimos 25 pacientes intervenidos el porcentaje descendió a $12 \%$. Este descenso se relacionó con la curva de aprendizaje, cambios tanto en la técnica quirúrgica, como en las técnicas empleadas en el banco de ojos y con la extensión del tiempo de llenado de la cámara anterior con aire a 45-60 minutos (22). En nuestra experiencia, el factor más importante para obtener una adhesión completa del tejido donante, es evitar el uso de ácido hialurónico durante todo el procedimiento (14). Al igual que en la DSEK, todos los viscoelásticos deberían ser evitados en DMEK, ya que el desprendimiento del tejido donante se correlaciona fuertemente con su utilización (14). Los experimentos in vitro han mostrado que un llenado con aire de la cámara anterior como mínimo de 30 minutos, es imprescindible para obtener una adhesión completa del tejido donante en DMEK, mientras que un llenado de aire de sólo 15 minutos sería suficiente en DLEK y DSEK $(8,14)$. 
Nuestra experiencia con DMEK, sugiere que el trasplante de MD donante aislada portadora de endotelio viable en el tratamiento de alteraciones del endotelio corneal, proporciona una restauración anatómica casi perfecta y un alto grado de rehabilitación visual. Al igual que en DLEK y DSEK, el mayor inconveniente del procedimiento, es el riesgo de desprendimiento del injerto en el curso postoperatorio precoz (16). Cuando el porcentaje de desprendimientos en DMEK pueda ser disminuido por una mejora, tanto en la logística del banco de ojos, como en la técnica quirúrgica, podría quedar finalmente completado el proyecto de las queratoplastias lamelares posteriores.

\section{BIBLIOGRAFÍA}

1. Melles GR, Eggink FA, Lander F, Pels E, Rietveld FJ, Beekhuis WH, et al. A surgical technique for posterior lamellar keratoplasty. Cornea 1998; 17: 618-626.

2. Melles GR, Lander F, Beekhuis WH, Remeijer L, Binder PS. Posterior lamellar keratoplasty for a case of pseudophakic bullous keratopathy. Am J Ophthalmol 1999; 127: 340-341.

3. Melles GR, Lander F, van Dooren BT, Pels E, Beekhuis WH. Preliminary clinical results of posterior lamellar keratoplasty through a sclerocorneal pocket incision. Ophthalmology 2000; 107: 1850-1857.

4. Terry MA, Ousley PJ. Deep lamellar endothelial keratoplasty in the first United States patients: early clinical results. Cornea 2001; 20: 239-243.

5. Melles GR, Lander F, Nieuwendaal C. Sutureless, posterior lamellar keratoplasty. Cornea 2002; 21: 325-327.

6. Terry MA, Ousley PJ. Small-incision deep lamellar endothelial keratoplasty (DLEK): six-month results in the first prospective clinical study. Cornea 2005; 24: 59-65.

7. Melles GR, Wijdh RH, Nieuwendaal CP: A technique to excise the descemet membrane from a recipient cornea (descemetorhexis). Cornea 2004; 23: 286-288.

8. Melles GR, Kamminga N. Tecniques for posterior lamellar keratoplasty through a scleral incision. Ophthalmologe 2003; 100: 689-695.

9. Price FW Jr, Price MO. Descemet's stripping with endothelial keratoplasty in 200 eyes: Early challenges and techniques to enhance donor adherence. J Cataract Refract Surg 2006; 32: 411-418.

10. Dapena I, Ham L, Melles GRJ. Endothelial keratoplasty. DSEK/DSAEK or DMEK: The thinner the better? Curr Opin Ophthalmol, in press.

11. Melles GRJ, Rietveld FJR, Pels E, Beekhuis WH, Binder PS. Transplantation of Descemet's membrane carrying viable endothelium through a small scleral incision. Invest Ophthalmol Vis Sci 1998; 39: S76.

12. Melles GR, Lander F, Rietveld FJ. Transplantation of Descemet's membrane carrying viable endothelium through a small scleral incision. Cornea 2002; 21: 415-418.
13. Melles GR, Ong TS, Ververs B, van der Wees J. Descemet membrane endothelial keratoplasty (DMEK). Cornea 2006; 25: 987-990.

14. Melles GR. Posterior lamellar keratoplasty: DLEK to DSEK to DMEK. Cornea 2006; 25: 879-881.

15. Melles GR, Ong TS, Ververs B, van der Wees J. Preliminary clinical results of Descemet membrane endothelial keratoplasty. Am J Ophthalmol 2008; 145: 222-227.

16. Ham L, van der Wees J, Melles GR. Causes of primary donor failure in Descemet membrane endothelial keratoplasty. Am J Ophthalmol 2008; 145: 639-644.

17. Nieuwendaal CP, Lapid-Gortzak R, van der Meulen IJ, Melles GJ. Posterior lamellar keratoplasty using descemetorhexis and organ-cultured donor corneal tissue (Melles technique). Cornea 2006; 25: 933-936.

18. Lie JT, Birbal R, Ham L, van der Wees J, Melles GR. Donor tissue preparation for Descemet membrane endothelial keratoplasty. J Cataract Refract Surg 2008; 34 : 1578-1583.

19. van Dooren BT, Mulder PG, Nieuwendal CP, Beekhuis WH, Melles GR. Endothelial cell density after deep posterior lamellar keratoplasty (Melles techniques): 3 years follow-up. Am J Ophthalmol 2004; 138: 211-217.

20. van Dooren BT, Mulder PG, Nieuwendal CP, Beekhuis $W H$, Melles GR. Endothelial cell density after deep anterior lamellar keratoplasty (Melles technique). Am J Ophthalmol 2004; 137: 397-400.

21. Tappin M. A method for true endothelial cell (Tencell) transplantation using a custom-made cannula for the treatment of endothelial cell failure. Eye 2007; 21: 775-779.

22. Ham L, Dapena I, van Luijk C, van der Wees J, Melles GR. Descemet membrane endothelial keratoplasty (DMEK) for Fuchs endothelial dystrophy: review of the first 50 consecutive cases. Eye. 2009. [Epub ahead of print]

23. Gorovoy MS. Descemet-stripping automated endothelial keratoplasty. Cornea 2006; 25: 886-889.

24. Holweger RR, Marefat B. Corneal changes after cataract surgery with $5.0 \mathrm{~mm}$ sutured and $3.5 \mathrm{~mm}$ sutureless clear corneal incisions. J Cataract Refract Surg 1997; 23: 342346.

25. Olsen T, Dam-Johansen M, Bek T, Hjortdal JO. Corneal versus scleral tunnel incision in cataract surgery: a randomized study. J Cataract Refract Surg 1997; 23: 337-341.

26. Lange TM, Wood TM, McLaughlin BJ. Corneal endothelial cell transplantation using Descemet's membrane as a carrier. J Cataract Refract Surg 1993; 19: 232-235.

27. Shimmura S, Miyashita H, Knomi K, Shinozaki N, Taguchi $T$, Kobayashi $H$, et al. Transplantation of corneal endothelium with Descemet's membrane using a hyroxyethyl methacrylate polymer as a carrier. Br J Ophthalmol 2005; 89: 134-137.

28. Ignacio TS, Nguyen TT, Sarayba MA, Sweet PM, Piovanetti $O$, Chuck RS, et al. A technique to harvest Descemet's membrane with viable endothelial cells for selective transplantation. Am J Ophthalmol 2005; 139: 325330.

29. Zhu Z, Rife L, Yiu S, Trousdale MD, Wasilewski D, Siqueira A, et al. A technique for preparation of the corneal endothelium-Descemet's membrane complex for transplantation. Cornea 2006; 25: 705-708. 
